\section{Application of innovative hemocytometric parameters and algorithms for improvement of microcytic anemia discrimination}

\author{
Margreet Schoorl, Marianne Schoorl, \\ Johannes van Pelt, Piet C.M. Bartels \\ Department of Clinical Chemistry, \\ Hematology and Immunology, Medical \\ Center Alkmaar, The Netherlands
}

\section{Abstract}

Hemocytometric parameters like red blood cell (RBC) count, mean red blood cell volume (MCV), reticulocyte count, red blood cell distribution width (RDW-SD) and zinc protoporphyrin (ZPP) are frequently established for discrimination between iron-deficiency anemia and thalassemia in subjects with microcytic erythropoiesis. However, no single marker or combination of tests is optimal for discrimination between iron-deficiency anemia and thalassemia. This is the reason why many algorithms have been introduced. However, application of conventional algorithms, only resulted in appropriate classification of $30-40 \%$ of subjects. In this mini-review the efficacy of innovative hematological parameters for detection of alterations in RBCs has been considered. It refers to parameters concerning hemoglobinization of RBCs and reticulocytes and the percentages microcytic and hypochromic RBCs, for discrimination between subjects with iron-deficiency anemia (IDA) or thalassemia as well as a combination of both. A new discriminating tool including the above mentioned parameters was developed, based on two precondition steps and discriminating algorithms. The percentage microcytic RBCs is considered in the first precondition step. MCV, RDW-SD and RBC count are applied in the second precondition step. Subsequently, new algorithms, including conventional as well as innovative hematological parameters, were assessed for subgroups with microcytic erythropoiesis. The new algorithms for IDA discrimination yielded results for sensitivity of $79 \%$, specificity of $97 \%$, positive and negative predictive values of $74 \%$ and $98 \%$ respectively. The algorithms for $\beta$-thalassemia discrimination revealed similar results (74\%, $98 \%, 75 \%$ and $99 \%$ respectively). We advocate that innovative algorithms, including parameters reflecting hemoglobinization of RBCs and reticulocytes, are integrated in an easily accessible software program linked to the hematology equipment to improve the discrimination between IDA and thalassemia.

\section{Introduction}

Anemia is a global public health problem affecting populations in both developing and developed countries. According to the World Health Organisation (WHO) anemia affects 1.62 billion people, which corresponds to approximately $25 \%$ of the world population. It is assumed that $50 \%$ of the cases of anemia are due to insufficient iron content in the diet, particularly in women in child-bearing age with increased menstrual blood loss or during pregnancy, young children and vegetarians. ${ }^{1}$

Iron-deficient erythropoiesis and thalassemia are both associated with mild to moderate microcytic anemia, which frequently results in an incorrect diagnosis.

It is important to discriminate between irondeficiency anemia (IDA) and thalassemia, in order to avoid unnecessary iron therapy and to prevent the development of hemosiderosis, which may result in serious complications like cardiomyopathy, liver fibrosis or endocrine dysfunctions. ${ }^{2,3}$

Regarding discrimination between iron deficiency and thalassemia, this mini-review focuses on the expedience of innovative hematological parameters concerning red blood cell (RBC) hemoglobinization as well as RBC production.

\section{Iron deficiency}

Nutritional deficiency as a result of inadequate iron, folic acid and vitamin B12 intake, will contribute to the development of anemia. Iron deficiency is considered to be the main cause for anemia: it is responsible for more than $50 \%$ of all cases. Iron deficiency results, amongst others, in impaired activity of several enzymes. As a consequence, iron depletion may result in serious health problems such as retarded growth, mental irritability, reduced resistance to infection and impaired intellectual development, particularly in case of infants in the growth phase. The causes for iron deficiency include decreased resorption of heme iron, increased consumption of phytate and phenolic compounds which inhibit iron absorption, increased requirement, and blood loss. ${ }^{4-6}$

According to WHO publications, the highest number of pre-school children, pregnant women and non-pregnant women suffering from IDA live in the Eastern Mediterranean countries, North-Africa and the Middle East. ${ }^{7}$ Despite the start of iron fortification, the prevalence of iron deficiency is still rather high in the Middle East countries. The prevalence of IDA in the Middle Eastern countries is equal to the prevalence in developing countries (25-35\%), which is much higher than in industrial countries (5-8\%). 8 ,9
Correspondence: Margreet Schoorl, Department of Clinical Chemistry, Hematology and Immunology, Medical Centre Alkmaar, Wilhelminalaan 12, 1815 JD Alkmaar, The Netherlands.

Tel.: +31.72.548.3625 - Fax: +31.72.548. 2175

E-mail: m.g.schoorl@mca.nl

Key words: Iron deficiency; thalassemia; anemia; erythropoiesis; reticulocyte hemoglobin.

Contributions: MgS, design of the study, intellectual content, drafted and revised the article; MiS, design of the study, intellectual content, comments to generate the final paper; JvP, reviewed the draft, comments to generate the final paper; PCMB, design of the study, intellectual content, drafted the article, comments to generate the final paper.

Conflict of interest: the authors declare no potential conflict of interest.

Received for publication: 2 February 2015.

Revision received: 6 May 2015

Accepted for publication: 29 May 2015.

This work is licensed under a Creative Commons Attribution NonCommercial 3.0 License (CC BYNC 3.0).

(C) Copyright M. Schoorl et al., 2015

Licensee PAGEPress, Italy

Hematology Reports 2015; 7:5843

doi:10.4081/hr.2015.5843

\section{Disorders of hemoglobin synthesis}

Hemoglobinopathy encompasses a group of genetic disorders which involve an abnormal structure of one of the globin chains of the hemoglobin molecule. In contrast, thalassemias are disorders of hemoglobin synthesis which usually results in decreased production of normal globin proteins, often due to mutations in regulatory genes. ${ }^{2,3}$

It is estimated that approximately $7 \%$ of the world's population carries mutations of globin genes. In several regions, the prevalence of thalassemia is even higher. With increasing migration of people, a genetic disease which was initially rare in northern Europe, Australia and North America is more common at the present time in these regions. The actual number of subjects with hemoglobinopathy and thalassemia worldwide is unknown and is probably underestimated. $3,7,10$

\section{Alpha-thalassemia}

Alpha-thalassemias are the most prevalent disorders of hemoglobin synthesis. ${ }^{3}$

Subjects with $\alpha$-thalassemia 2 heterozygos- 
ity (with three functional $\alpha$-globin genes: $\alpha \alpha /$ $\alpha$ ) are characterized by normal to moderately decreased mean cell volume (MCV) values with normal or slightly decreased hemoglobin concentrations. ${ }^{11,12}$ Subjects with homozygous $\alpha$-thalassemia $2(-\alpha /-\alpha)$ and heterozygous $\alpha$ thalassemia $1(\alpha \alpha /--)$ are phenotypically very similar and cannot be distinguished by only blood count markers. Erythropoiesis with decreased MCV, frequently associated with mild anemia, is frequently observed.

\section{Beta-thalassemia}

Mutations causing $\beta$-thalassemia result in a lack of $\beta$-globin production, which ranges from minimal (mild $\beta$--thalassemia alleles) to a complete absence ( $\beta 0$-alleles).

Almost all heterozygous conditions (except the so-called normal HbA2-thalassaemias, e.g. $\delta \beta$-thalassemia), are characterized by an increased $\mathrm{HbA} 2$ content $(\geq 3.2 \%)$ and a marked degree of microcytosis, which is frequently associated with mild anemia. Subjects with homozygous $\beta$-thalassemia suffer from severe microcytic anemia.

\section{Hemoglobinopathies}

Hemoglobinopathies encompass a heterogeneous group of inherited disorders which affect the structure of the hemoglobin molecule, resulting in microcytic erythropoiesis. Four abnormal hemoglobins ( $\mathrm{Hb})$, in particular $\mathrm{HbS}, \mathrm{HbC}, \mathrm{HbE}$ and $\mathrm{HbD}$ are rather common in various parts of the world, e.g. Africa, the Mediterranean area and Southeast Asia. Carriers of hemoglobinopathies, if not associated with $\alpha$-thalassemia, usually do not suffer from anemia while results of MCV and mean red blood cell hemoglobin (MCH) are situated in the lower part of the reference range.

\section{Discrimination between iron deficiency and thalassemia syndromes}

The discrimination between IDA and thalassemia has important clinical implications. A reliable diagnosis is important in order to reduce unnecessary laboratory testing and to avoid inappropriate treatment. A wide range of laboratory parameters is available to facilitate the discrimination between IDA and thalassemia. However, no single marker or combination of tests is optimal for discrimination between iron deficiency and thalassemia. In addition, iron deficiency often occurs in combination with other diseases which complicates the differential diagnosis. Diagnosing subjects with combined thalassemia minor and iron deficiency is even more challenging.

In order to facilitate the discrimination pro- cedure a number of algorithms have been described, which combine routine RBC parameters and indices obtained from routine complete blood count. At least 40 of these algorithms are published. Well known conventional algorithms are those of Mentzer, England\&Fraser, Green\&King, Shine\&Lal, Ricerca and Shrivastava (Table 1). ${ }^{13-18}$ However, none of these algorithms is appropriate for a correct classification. For example, the algorithms of Mentzer, England\&Fraser and Green\&King only resulted in appropriate classification of $30-40 \%$ of subjects with $\beta$-thalassemia.

In the late $80 \mathrm{~s}$, the determination of ZPP in $\mathrm{RBC}$ was recommended to appropriately discriminate subjects with iron deficiency from subjects with thalassemia. ZPP is a biomarker for the detection of long term iron-deficient erythropoiesis. ${ }^{19}$ ZPP results are obviously increased in patients with iron deficiency and, to a lesser degree, in subjects with $\alpha$ - or $\beta$-thalassemia trait. ${ }^{20-22}$ In addition, introduction of discriminating algorithms including MCV, $\mathrm{MCH}, \mathrm{RBC}$ and red blood cell distribution width (RDW) has advanced the differential diagnosis of iron deficiency and $\alpha$ - or $\beta$-thalassemia, but in several cases these algorithms still resulted in an inappropriate diagnosis. ${ }^{23}$

Over the past decade, discriminating procedures and algorithms have been improved stepby-step.

In 2003 the efficacy of a discriminating algorithm including the hematological parameters RBC, RDW-SD, ZPP and reticulocyte count has been established. ${ }^{24} \mathrm{MCV}$ is recommended as an initial test. Subsequently, appropriate classification was achieved in $90 \%$ of the subjects with iron deficiency, $\alpha$-thalassemia or $\beta$-thalassemia by application of the algorithm $2 \times \mathrm{RDW}(\mathrm{fL})-5 \times \mathrm{RBC}\left(\times 10^{12} / \mathrm{L}\right)-250 \times$ reticulocytes $\left(\times 10^{12} / \mathrm{L}\right)+30 \times \mathrm{ZPP}(\mu \mathrm{mol} / \mathrm{mol} \mathrm{Hb}){ }^{24}$

The introduction of new generations of hematology equipment enabled the use of innovative hemocytometric parameters for hemoglobinization in reticulocytes and RBCs. The reticulocyte hemoglobin content $(\mathrm{CHr})$ was initially determined on the Bayer $\mathrm{H}^{*} 3$ hematology analyzer. ${ }^{25}$ The $\mathrm{CHr}$ is determined from measurements of light scatter at two different angles after isovolumetric sphering of oxazine-750 stained reticulocytes. ${ }^{25,26} \mathrm{~A}$ few years later Sysmex Corporation (Tokyo, Japan) introduced parameters indicating the hemoglobin content of reticulocytes and RBCs, RET$\mathrm{He}$ and $\mathrm{RBC}-\mathrm{He}$ respectively. The hemoglobin content of RBCs and reticulocytes is established by means of the flow cytometric methodology. The mean forward light scattered intensity in the reticulocyte channel is measured as a parameter for estimation of the cell volume and to establish simultaneously hemoglobin content of RBCs and reticulocytes. Ret-He has demonstrated to be comparable with $\mathrm{CHr}^{27}$

Hemoglobin content in reticulocytes reflects a short term indication of the functional availability of iron for erythropoiesis and the response to iron supplementation. Reticulocyte maturation occurs with progressive decrease in RBC volume and hemoglobin content. If compared with interpretation of $\mathrm{Hb}$ content of mature RBCs, interpretation of $\mathrm{Hb}$ content of reticulocytes yields additional information concerning decreased functional availability of iron for hemoglobinization of erythroid precursors. ${ }^{28,29}$

Thomas et al. introduced a diagnostic model including the hemoglobin content of reticulocytes in combination with the soluble transferrin receptor/log ferritin ratio (sTfR-F index) for the purpose of monitoring the progression of iron deficiency, regardless of acute phase response. $^{28}$

In 2006 the additional value of the innovative hematological parameters in case of subjects with IDA and thalassemia has been explored. Ret-He and RBC-He results were decreased in the subgroups with IDA and $\alpha$ - or $\beta$-thalassemia, if compared with the group of apparently healthy subjects. For the Ret$\mathrm{He} / \mathrm{RBC}-\mathrm{He}$ ratio obviously decreased results were demonstrated in subjects with IDA $(1.02 \pm 0.08$, mean \pm SD) and to a lesser extent in subjects with $\beta$-thalassemia $(1.06 \pm 0.04)$ if compared with the group of healthy reference subjects $(1.11 \pm 0.02)$ and subjects with $\alpha$-thalassemia $(1.11 \pm 0.07)$. A combination of results

Table 1. Overview of discrimination formulas including cut-off levels for iron deficiency anemia and for $\beta$-thalassemia.

\begin{tabular}{|c|c|c|c|}
\hline Authors & Formulas & Iron deficiency anemia & Thalassemia \\
\hline England \& Fraser ${ }^{14}$ & MCV - RBC - $5 \times \mathrm{Hb}-3.4)$ & $>0$ & $<0$ \\
\hline Green \& King 15 & $\left(\mathrm{MCV}^{2} \times \mathrm{RDW}-\mathrm{CV}\right) /(\mathrm{Hb} \times 100)$ & $>65$ & $<65$ \\
\hline Mentzer ${ }^{13}$ & $\mathrm{MCV} / \mathrm{RBC}$ & $>13$ & $<13$ \\
\hline Shine $\&$ Lal16 & $M C{ }^{2} \times M C H \times 0.01$ & $>1530$ & $<1530$ \\
\hline Ricerca17 & RDW/RBC & $>4.4$ & $<4.4$ \\
\hline Shrivastava ${ }^{18}$ & $\mathrm{MCH} / \mathrm{RBC}$ & $>3.8$ & $<3.8$ \\
\hline
\end{tabular}

$M C V$, mean red blood cell volume; $\mathrm{RBC}$, red blood cell; $\mathrm{Hb}$, hemoglobin; $\mathrm{RDW}-\mathrm{CV}$, red blood cell distribution width; $\mathrm{MCH}$, mean red blood cell hemoglobin. 
for Ret-He, Ret-He/RBC-He ratio in relation to MCV results yields an appropriate method for obtaining insight into the degree of hemoglobinization and RBCs characteristics for proper clinical interpretation in the case of subjects with IDA or thalassemia. Cut-off levels for Ret$\mathrm{He}$ of $<29.5 \mathrm{pg}$ and for Ret-He/RBC-He ratio of $<1.02$ are advocated as indicators of iron-deficient erythropoesis. ${ }^{30}$

More recently, innovative methods for establishment of microcytic and hypochromic RBCs have been introduced. Measurement of microcytic and hypochromic RBCs was first available on Technicon hematology analyzers (currently Advia ${ }^{\circledR}$, Siemens Medical Solutions, Malvern, USA), applicating multidimensional light scatter by sphered RBCs, following the Mie theory principles. ${ }^{31-33}$ Already in 1992 d'Onofrio et al. reported on the ratio between the percentage of microcytes and the percentage of hypochromic cells, the so-called M/H-ratio. ${ }^{34}$ Iron-deficient erythropoiesis is characterized by the production of RBCs with a severely decreased $\mathrm{Hb}$ concentration, while microcytic RBCs in subjects with $\beta$-thalassemia are generally smaller, with a more preserved $\mathrm{Hb}$ concentration. In subjects with $\beta$-thalassemia, the percentage of microcytic RBCs is higher than the percentage of hypochromic RBCs. On the contrary, in subjects with IDA, the percentage of hypochromic RBCs is higher than the percentage of microcytic RBCs. ${ }^{34}$ Measurement of the percentage hypochromic and microcytic RBCs, has demonstrated to be useful for detection of rather small changes in the amount of RBCs with inadequate haemoglobinisation. ${ }^{28,35,36}$

In 2008 Urrechaga et al. reported on improved discriminating formulas including the percentage hypochromic and microcytic RBCs (indicated as \%Hypo-He and \%MicroR respectively) combined with RDW-CV. ${ }^{37}$

The parameter \%HypoHe reflects the percentage of hypochromic RBCs with a Hb content of less than $17 \mathrm{pg}$. \%MicroR indicates the percentage of microcytic RBCs with a volume of less than $60 \mathrm{fL} .37,38$

In cases of $\beta$-thalassemia, obviously increased results for \%MicroR are observed (mean \pm SD, 37.8 \pm 11.4 ) if compared with the groups of healthy subjects $(1.1 \pm 0.44)$ and subjects with IDA $(19.1 \pm 9.7)$. In cases of IDA, results of \%Hypo-He are increased (mean $\pm \mathrm{SD}$, $15.7 \pm 11.5$ ) compared with the groups of healthy subjects $(0.3 \pm 0.16)$ and subjects with $\beta$-thalassemia (11.9 \pm 7.2$)$. In the same study, the efficacy of application of algorithms is explored such as \%MicroR minus \%Hypo-He (M-H). The cut off value which provides the best results for application of Youden index for M-H was 11.5 ( $\beta$-thalassemia $>11.5$, IDA<11.5). ${ }^{37,38}$

An European multicenter study established the additional value of new discriminating algorithms which include the parameters for hemoglobinization of reticulocytes (Ret-He), Delta-He (RET-He minus RBC-He) and the percentages of microcytic RBCs (\%MicroR) and hypochromic RBCs (\%HypoHe) for discrimination between IDA and $\beta$-thalassemia. ${ }^{39}$ The study established the sensitivity and specificity of the novel discriminating algorithms between these groups and a control group of healthy subjects and compared the algorithms with currently available algorithms for discrimination. To illustrate the decision making process for discriminating between IDA and $\beta$-thalassemia, pre-selection criteria and algorithms are depicted in Table 2. Application of the new algorithms for discriminating IDA yielded in an area under the curve 0.88 , a sensitivity of $79 \%$, a specificity of $97 \%$ and positive and negative predictive values of $74 \%$ and $98 \%$, respectively. For the detection of $\beta$-thalassemia similar results were achieved $(0.86,74 \%, 98 \%, 75 \%$, and $99 \%$, respectively). ${ }^{39}$ Evaluation of the diagnostic efficacy of the algorithms demonstrated excellent performance, if compared with conventional discriminating algorithms described by England\&Fraser, Green\&King and Mentzer. ${ }^{13-}$ 15 Nevertheless, none of the algorithms provided $100 \%$ sensitivity and $100 \%$ specificity in discrimination between subjects with iron deficiency and subjects with $\beta$-thalassemia. Therefore, after screening with the six algorithms, confirmatory testing should be performed for proper diagnosis. ${ }^{39}$

\section{Anemia discrimination in subjects with combinations of iron-deficiency anemia and thalassemia}

When facing the challenge of a discriminating algorithm for subjects with combinations of $\beta$-thalassemia and IDA, application of Ret$\mathrm{He}$ and Ret-He/RBC-He ratio as additional parameters is recommended. In this particular group of subjects, Ret-He and Ret-He/RBC-He ratio are obviously decreased (respectively $29.5 \mathrm{pg}$ and <1.02)..$^{30}$

In subjects with combinations of $\beta$-thalassemia and IDA, also a combination of \%Hypo-He and $\mathrm{M}-\mathrm{H}$ index seems to be promising. ${ }^{40}$ In this group of subjects markedly increased \%Hypo-He $(>20)$ occur in combination with a decreased M-H index $(<11.5)$ (personal communication). Further investigation is required.

\section{Critical remark}

Several manufacturers of automated hematology equipment facilitate establishment of innovative parameters like hemoglobin content of RBCs and reticulocytes. However, the comparability of these parameters is a matter of serious concern. Particularly in multicenter-settings, calibration and adjustment of the measuring channels is an important issue. In case of multicenter evaluation studies involving hematology equipment from different suppliers, validation and mutual alignment is required.

\section{Conclusions}

It is strongly advised to use discriminating algorithms as a tool for anemia discrimination in order to reduce diagnostic testing for confir-

Table 2. Preconditions and algorithms for discrimination microcytic anemia on iron deficiency anemia (IDA) and beta-thalassemia (THAL).

\begin{tabular}{|c|c|c|c|}
\hline Precondition 1 & Precondition 2 & Algorithm & \\
\hline$\% M i c r o R \geq 3$ & $M C V<85$ and $>=75$ AND $\% M i c r o R \geq 5$ & \%MicroR / \%HypoHe <4 AND [( MCV² x RDW-CV) / (Hb*100)] $\geq 75$ AND Reti\#<0.08 & IDA \\
\hline$\% M i c r o R \geq 3$ & $M C V<75$ and $\geq 65$ & \%MicroR / \%HypoHe <3.4 AND [( MCV2 x RDW-CV) /(Hb*100) ] $\geq 77$ AND Reti\#<0.08 & IDA \\
\hline$\% M i c r o R \geq 3$ & $M C V<65$ & \%MicroR - \%HypoHe - RDW-CV $<-5.2$ & IDA \\
\hline$\% M i c r o R \geq 3$ & $\begin{array}{l}M C V<85 \text { and } \geq 75 \text { AND RDW- } \\
S D<44.7 \text { AND RBC } \geq 3.50\end{array}$ & $\begin{array}{c}{\left[\left(\text { MCV } \times \text { RDW-CV) } /\left(\mathrm{Hb}^{*} 100\right)\right]<75 \text { AND [ ( MCV-RBC }-3.4-\left(5^{*} \mathrm{Hb}\right)\right]} \\
<4 \text { AND Reti } \# 0.08 \text { AND Delta-He } \geq 0\end{array}$ & THAL \\
\hline$\% M i c r o R \geq 3$ & $M C V<75$ and $\geq 65$ AND RBC $\geq 3.50$ & {$\left[(\mathrm{MCV} 2 \times \mathrm{RDW}-\mathrm{CV}) /\left(\mathrm{Hb}^{*} 100\right)\right]<77$ AND \%MicroR / \%HypoHe $\geq 2.0$} & THAL \\
\hline$\% M i c r o R \geq 3$ & $M C V<65$ & (\%MicroR - \%HypoHe - RDW-CV) $\geq-5.2$ & THAL \\
\hline
\end{tabular}

\%MicroR, percentage microcytic red blood cells; MCV, mean red blood cell volume; \%HypoHe, percentage hypochromic red blood cells; RDW, red blood cell distribution width. Parameters used in the algorithms are expressed in the following units: red blod cells (1012/L), hemoglobin ( $g / d L), M C V(f L), \operatorname{RDW}-S D(f L), \operatorname{RDW}-C V(\%), \operatorname{Reti} \#\left(10^{12} / L\right)$. 
mation and to properly diagnose the underlying cause(s) in the patients.

It is advocated that innovative algorithms, including parameters reflecting hemoglobinization of RBCs and reticulocytes, are integrated in an easily accessible software program linked to the hematology equipment, preferably provided by the manufacturer of the equipment to facilitate this interaction, to improve the discrimination between IDA and thalassemia.

\section{References}

1. WHO. World prevalence of anaemia 19932005. WHO Global database on anaemia. Available from: apps.who.int/iris/bitstream/10665/43894/1/9789241596657_en g.pdf?ua $=1$.

2. Weatherall DJ, Clegg JB. The thalassaemia syndromes. $4^{\text {th }}$ ed. Oxford: Blackwell Science; 2001.

3. Higgs DR, Engel JD, Stamatoyannopoulos G. Thalassaemia. Lancet 2012;379:373-83.

4 Fairbanks VF, Beutler E. Iron deficiency. In: Beutler E, Lichtman MA, Coller B, et al, eds. Williams hematology. $6^{\text {th }}$ ed. New York: Mc Graw-Hill; 2001. pp 447-470.

5 Hurrell R, Ranum P, de Pee S, et al. Revised recommendations for iron fortification of wheat flour and an evaluation of the expected impact of current national wheat flour fortification programs. Food Nutr Bull 2010;31:S7-21.

6. WHO. Research for universal health coverage: world health report 2013. Available from: http://www.searo.who.int/indonesia/ documents/research-for-universal-healthcoverage\%289789240690837_eng\%29.pdf

7. Modell B, Darlison M. Global epidemiology of haemoglobin disorders and derived service indicators. Bull World Health Organ 2008;86:480-7.

8. Stolzfus RJ. Iron deficiency: global prevalence and consequences. Food Nutr Bull 2003;24:S99-103.

9. Mirmiran P, Golzarand M, Serra-Majem L, Azizi F. Iron, iodine and vitamin A in the Middle East: a systematic review of deficiency and food fortification. Iranian $\mathrm{J}$ Publ Health 2012;41:8-19.

10. Weatherall DJ. The inherited diseases of haemoglobin are an emerging global health burden. Blood 2010;115:4331-6.

11. Maude GH, Higgs DR, Beckford M, et al. Alpha thalassaemia and the haematology of normal Jamaican children. Clin Lab Haematol 1985;7:289-95.
12. Pornpatkul M, Wasi P, Na-Nakorn S. Haematologic parameters in obligatory alpha thalassaemia traits. J Med Assoc Thai 1969;52:801-9.

13. Mentzer WC Jr. Differentiation of iron deficiency from thalassaemia trait. Lancet 1973;1:882.

14. England JM, Fraser PM. Differentiation of iron deficiency from thalassaemia trait by routine blood count. Lancet 1973;1:449-52.

15. Green R, King R. Red cell discriminant function, $\beta$-thalassaemia minor versus iron deficiency. Blood Cells 1989;15:481-95.

16. Shine I, Lal S. A strategy to detect beta-thalassemia minor. Lancet 1977;1:692-4.

17. Ricerca BM, Storti S, d'Onofrio G, et al. Differentiation of iron deficiency from thalassaemia trait: new approach. Hematologica 1987;72:409-13.

18. Srivastava PC, Bevington JM. Iron deficiency and/or thalassaemia trait. Lancet 1973;1:832

19. Blumberg WE, Eisinger J, Lamola AA, et al. Principles and applications of haematofluorometry. J Clin Lab Autom 1984;4:29-42.

20. Han P, Fung KP, Teo CP, et al. Alpha-thalassaemia and beta-thalassaemia traits: biological difference based on red cell indices and zinc protoporphyrin. Clin Lab Haematol 1990;12:169-76.

21. Harthoorn-Lasthuizen EJ, Lindemans J, Langenhuijsen MM. Combined use of erythrocyte zinc protoporphyrin and mean corpuscular volume in differentiation of thalassaemia from iron deficiency anemia. Eur J Hematol 1998;60:245-51.

22. Labbé RF, Vreman HJ, Stevenson DK. Zinc protoporphyrin: a metabolite with a mission. Clin Chem 1999;45:2060-72.

23. Eldibany MM, Totonchi KF, Joseph NJ, et al. Usefulness of certain red blood cell indices in diagnosing and differentiating thalassaemia trait from iron deficiency. Am J Clin Pathol 1999;111:676-82.

24. Bartels PCM, Schoorl M, Schoorl M. Application of haemocytometry in combination with reticulocytes count and red cell zinc protoporphyrin for appropriate classification of microcytic anaemia. Sysmex J Int 2003;13:78-82.

25. D'Onofrio G, Chirillo R, Zini G, et al. Simultaneous measurement of reticulocyte and red blood cell indices in healthy subjects and patients with microcytic and macrocytic anemia. Blood 1995;85:818-23.

26. Brugnara C, Laufer MR, Friedman AJ, et al. Reticulocyte hemoglobin content (CHr): early indicator of iron deficiency and response to therapy (letter). Blood 1994; 83:3100-1.

27. Thomas L, Franck S, Messinger M, et al.
Reticulocyte haemoglobin measurement comparison of two methods in the diagnosis of iron-restricted erythropoiesis. Clin Chem Lab Med 2005;43:1193-202.

28. Thomas C, Thomas L. Biochemical markers and hematologic indices in the diagnosis of functional iron deficiency. Clin Chem 2002;48:1066-76.

29. Brugnara C. Iron deficiency and erythropoiesis: new diagnostic approaches. Clin Chem 2003;49:1573-8.

30. Bartels PCM, Schoorl M, Schoorl M. Hemoglobinization and functional availability of iron for erythropoiesis in case of thalassemia and iron deficiency anemia. Clin Lab 2006;52:107-14.

31. Kim YR, Ornstein L. Isovolumetric sphering of erythrocytes for more accurate and precise cell volume measurement by flow cytometry. Cytometry 1983;3:419-27.

32. Tycko DH, Metz MH, Epstein EA, Grinbaum A. Flow-cytometric light scattering measurement of red blood cell volume and hemoglobin concentration. Appl Opt 1985; 24:1355-65.

33. Mohandas N, Kim YR, Tycko DH, et al. Accurate and independent measurement of volume and hemoglobin concentration of individual red cells by laser light scattering. Blood 1986;68:506-13.

34. d'Onofrio G, Zini G, Ricerca BM, et al. Automated meausument of red blood cell microcytosis and hypochromia in iron deficiency and $\beta$-thalassemia trait. Arch Pathol Lab Med 1992;116:84-9.

35. Navarro JF, Macia ML. Hypochromic red cells as an indicator of iron deficiency. $\mathrm{J}$ Rheumatol 1997;24:804-5.

36. Macdougall IC. Merits of hypochromic red cells as a marker of functional iron deficiency. Nephrol Dial Transplant 1998;13:8 47-9.

37. Urrechaga E. Discriminant value of $\%$ microcytic/\%hypochromic ratio in the differential diagnosis of microcytic anemia. Clin Chem Lab 2008;46:1752-8.

38. Urrechaga E, Borque L, Escanero F. The role of automated measurement of $\mathrm{RBC}$ subpopulations in differential diagnosis of microcytic anemia and $\beta$-thalassaemia. Am J Clin Pathol 2011;135:374-9.

39. Schoorl M, Schoorl M, Linssen J, et al. Efficacy of advanced discriminating algorithms for screening on iron-deficiency anemia and $\beta$-thalassemia trait. Am J Clin Pathol 2012;138:300-4.

40. Schoorl M. Combined iron deficiency and thalassemia minor. Author reply on comment Burdick C. Am J Clin Pathol 2013; 139;259-62. 